\title{
CFD Simulation Analysis for the Wax-type Thermostat
}

\author{
Lang Bai \\ Mechanical and Precision Instrument Engineering \\ Xi'an University of Technology \\ Xi'an 710048, China \\ E-mail:bailangdyx@163.com \\ Mingshun Yang \\ Mechanical and Precision Instrument Engineering \\ Xi'an University of Technology \\ Xi'an 710048, China
}

\author{
Yan $\mathrm{Li}$ \\ Mechanical and Precision Instrument Engineering \\ Xi'an University of Technology \\ Xi'an 710048, China
}

\begin{abstract}
Thermostat is an important part of car cooling system. In this paper, with the fluid analysis software FLUENT, CFD simulation analysis was carried out on the two kinds of thermostat. The distributions of temperature, speed, pressure were obtained by simulation analysis of flow field of thermostat, and the internal fluid flow state and its change law of thermostat were obtained under different opening. One of thermostat with the warm wind pipe was simulated and implemented a simple optimization, finally. Through the simulation in the flow field of the thermostat at different opening, it could be obtained that the thermostat internal fluid flow state and its variation in different operating conditions. It had important significance on the thermostat working law prediction and the thermostat cooling system performance analysis. The state of the coolant through the thermostat inner was reflected clearly and intuitively by the visual graphics of flow field inside the thermostat with the CFD simulation analysis. The structure of the thermostat could be appropriately improved or optimized by the temperature, pressure, turbulent kinetic energy and other factors.
\end{abstract}

Keywords-thermostat; FLUENT; flow visualization; $3 D$ model; simulation

\section{INTRODUCTION}

Temperature regulator, also known as thermostat, installed in the water outlet or water intake of the cooling system, could automatically adjust the flow direction and flux of cooling fluid according to the water temperature of installation position, maintaining the working temperature of engine in the set range, to prevent the engine from being too hot or too cold. The performance of the thermostat plays an important role in the performance of engine and cooling system.

The current study on related field mainly focuses on the intelligent development. Zhiheng Chen developed a new type of memory alloy thermostat; Peng Liu carried out the research of pneumatic type thermostat [1]; Shuai Wang used the electric tee proportional valve to replace the traditional mechanical type thermostat in the cooling system to realize the intelligent electronic control on the thermostat; $\mathrm{T}$. Mitchen used the wax-type thermostat, electric two-way valve, electric three-way valve and no thermostat four

cases to respectively carry out the engine warming up experiments [2]; Wuhan University of Technology, Huazhong University of Science and Technology and Dongfeng commercial vehicle technical center together researched a type of thermostat, a new type of cooling system synchronously electrically controlled by fan and water pump, which realized the joint intelligent electronic control parameter sharing and complementary among the thermostat, fan and water pump, which improved the overall performance of the cooling system.

Due to the good reliability and high cost performance, wax-type thermostat still occupies absolute advantage in car engine, especially in the application of commercial vehicle engine [3]. Because of the big market demand, the simple principle, the work which is not influenced by water pressure of engine cooling system, the small flow resistance, and the long service life, so wax-type thermostat is difficult to be replaced in a short period of time [4]. The mechanical structure and the working principle of valves decide that wax-type thermostat has a great space in the improvement of structure and function. Using the finite element method in the flow field numerical simulation analysis of thermostat to realize visualization of the internal of thermostat, it has important theoretical and practical engineering significance in optimizing the channel, enhancing the work performance and increasing the cooling capacity of the cooling system [5].

Current research on the wax-type thermostat is rare. Because it belongs to the fluid components, method of CFD on flow field can achieve the purpose of the analysis of flow characteristics and the improvement of 
structure. This paper attempts to use fluid analysis software FLUENT to conduct CFD simulation analysis of the mask body thermostat. First, the flow fields of thermostat models were analyzed at four different opening, which can get its flow field velocity, pressure and temperature distribution, then can obtain the fluid flow state and its variation law inside the thermostat at different boundary conditions. It had important significance to predict the thermostats work rules and to analyze its performance. On this basis, a thermostat with a heater tubes were analyzed. It is simply optimized through the flow field distribution figure.

\section{SIMULATION MODEL BUILDING}

\section{A. Establishment of 3D Model}

According to the thermostat model provided by the enterprise, the establishment of the flow field model by $\mathrm{PRO} / \mathrm{E}$, and the flow field model of the no warm air pipeline thermostat were established. We extended appropriately the import and export of flow field model of the thermostat to ensure full flow so that the convergence was more easily in the calculation. Ignoring the spring and portion of stents in order to simplify the model during modeling. To establish the opening of flow field model of thermostat by $\mathrm{PRO} / \mathrm{E}$ (lift of the main valve is $3.5 \mathrm{~mm}$ ), as shown in Fig.1.

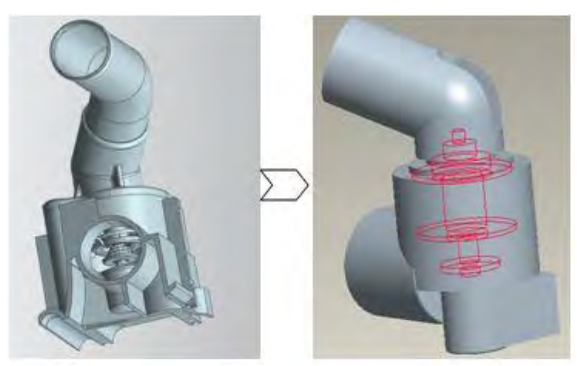

Figure 1. Establishment of model

\section{B. Mesh Generation}

Before the simulation analysis, we needed to mesh the model. Due to the asymmetric flow field model, so the mesh should be carried out for the entire threedimensional flow field. Converted the model of flow field established by PRO/E to general format of IGES, and then imported the model into the mesh software GAMBIT for meshing. When generated mesh, we extended appropriately the import and export of field model of the thermostat to ensure full flow [6]. In this paper, the mesh type is mainly tetrahedron. The structure of temperature-sensitive area of the center is complex, where the mesh was refined. The more number of meshes generated, the more great amounts of calculation and the more accurate analysis results. The number of meshes is 233181, and the model of meshes is as shown in Fig .2.

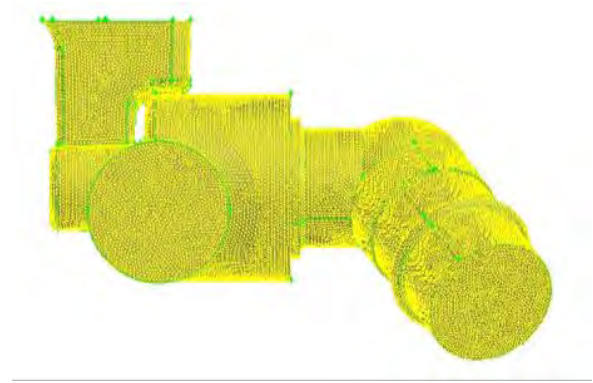

Figure 2. Mesh generation of model

\section{SIMULATION ANALYSES}

\section{A. The Basic Theory}

The fluid medium within thermostat was assumed reasonably as incompressible viscous fluid in the course of the study, with water as the calculation of the medium. The control equation of incompressible viscous fluid consists of the mass conservation equation (continuity equation), the momentum conservation equations (N-S equations) as well as the energy conservation [7], namely:

1) The mass conservation equation (continuity equation)

$$
\frac{\partial \rho}{\partial t}+\frac{\partial(\rho u)}{\partial x}+\frac{\partial(\rho v)}{\partial y}+\frac{\partial(\rho w)}{\partial z}=0
$$

$\rho$ is the fluid density. $\mathrm{t}$ is the time. $\mathrm{u}, \mathrm{v}$ and $\mathrm{w}$ are the velocity components of velocity vector on the three coordinate axes. For the incompressible fluid, the fluid density $\rho$ is constant. The equation became:

$$
\frac{\partial u}{\partial x}+\frac{\partial v}{\partial y}+\frac{\partial w}{\partial z}=0
$$

2) The momentum conservation equations (N-S equations)

$$
\begin{gathered}
\frac{\partial(\rho u)}{\partial t}+\operatorname{div}(\rho u V)=\operatorname{div}(\mu \operatorname{grad} u)-\frac{\partial p}{\partial x}+S_{u} \\
\frac{\partial(\rho v)}{\partial t}+\operatorname{div}(\rho v V)=\operatorname{div}(\mu \operatorname{grad} v)-\frac{\partial p}{\partial y}+S_{v} \\
\frac{\partial(\rho w)}{\partial t}+\operatorname{div}(\rho w V)=\operatorname{div}(\mu \operatorname{grad} w)-\frac{\partial p}{\partial z}+S_{w}(3)
\end{gathered}
$$

Where, $p$ is the pressure on the fluid micro unit. $S_{u} 、 S_{v}$ and $S_{w}$ are the generalized source terms for the momentum conservation equation.

3) The energy conservation (first law of thermodynamics)

$$
\frac{\partial(\rho T)}{\partial t}+\operatorname{div}(\rho V T)=\operatorname{div}\left(\frac{k}{c_{p}} \operatorname{grad} T\right)+S_{T}(4)
$$

\section{B. Simulation Conditions}

The initial conditions of simulation are as follows: the temperature and flow of a water outlet of the engine had been known, the temperature is determined by a given opening, the flow rate and the temperature of coolant were respectively calculated in the large and small cycle, the basic solving equation was the threedimensional incompressible N-S equations, the standard 
k- $\varepsilon$ model used in the turbulence model, the wall function used in the near wall region, the SIMPLE algorithm on unstructured meshes used in the solution of discrete equations [8], the implicit whole field iterative methods used in the velocity and pressure field, the boundary conditions determined inlet velocity and outlet pressure [9].

\section{The simulation results}

The internal flow field was observed through an appropriate observation face established after the simulation. We got the distribution of temperature, pressure and speed in the internal section of thermostat under different opening and the temperature threedimensional figures of the temperature sensing body of thermostat [10], as shown in Fig .3 and Fig .4.
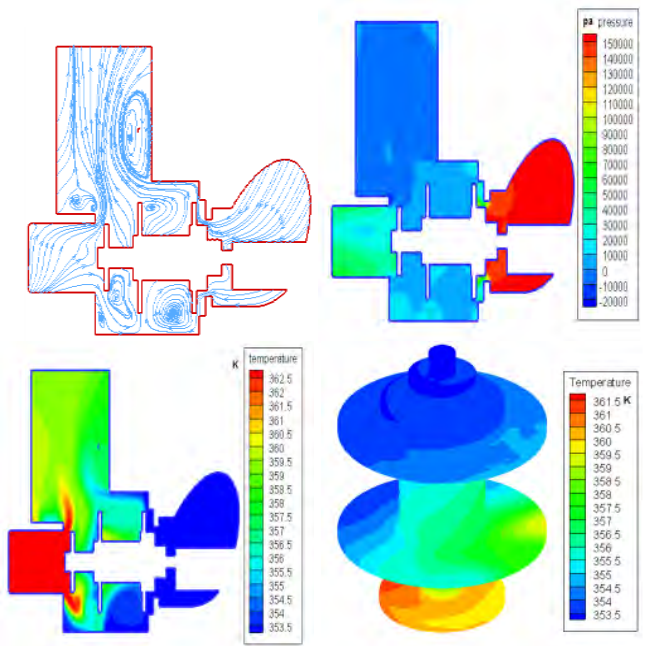

Figure 3. The flow field distribution of one opening of thermostat

Through observing the simulation results of flow field of thermostat, we could see the state of the cooling fluid flowing through the thermostat. We could get the area of the high-pressure and low-pressure which generated during the thermostat work through pressure figure. And we could get the temperature distribution in the vicinity of the temperature sensing body of thermostat and the temperature of thermostat outlet through the temperature distribution figure. It played an important role in the determination of function to be good or bad and in the optimization of its structure.
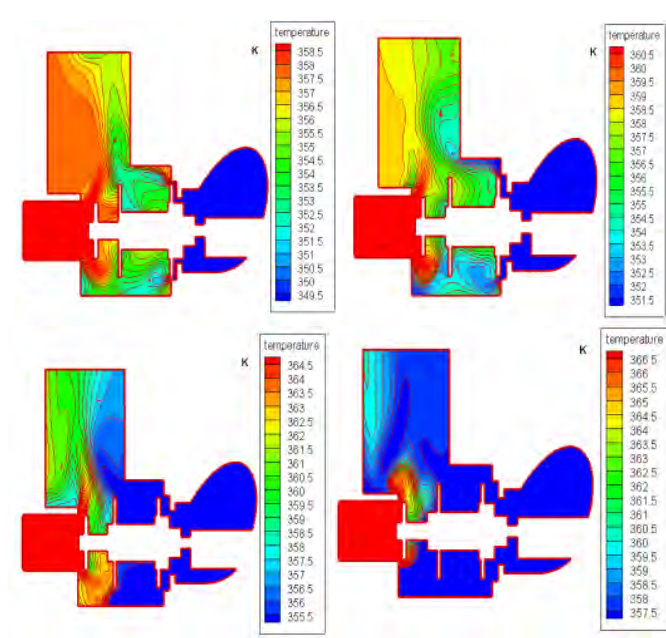

Figure 4. The flow field sectional temperature distributions of four different openings of the thermostat

After the fluid temperature of the outlet being detected, we obtained an average temperature variation at the outlet in different opening, shown in the Fig .5. When the engine started the job, because the system temperature was low and the opening of main valve of the thermostat was relatively small, the temperature of the hot water mixing with cold water was still relatively low. With the increase of its thermal load, the system temperature increased gradually, the opening of main valve of thermostat became larger. With the increase of cold water flow and the reduction of hot water flow, the mixed outlet temperature would reach the maximum at about $50 \%$ opening. With the system temperature continued to rise, the main valve of thermostat continued to become larger and cold water was becoming more and more, the mixed outlet temperature reduced gradually to the minimum [11].

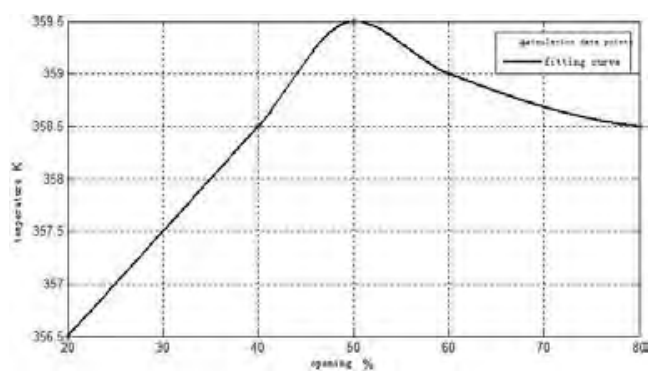

Figure 5. The relationship between outlet temperature and opening

The model of mask body thermostat used above was without the warm wind pipe. If coupled with the warm wind pipe, the model would change and the internal of flow field would also change. A thermostat with $50 \%$ opening was simulated by CFD, as follow: 


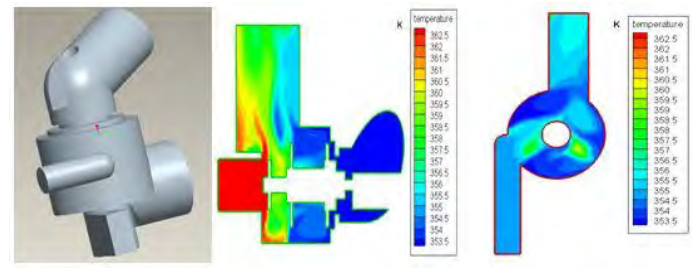

Figure 6. The model of thermostat with the warm wind pipe and the temperature distribution

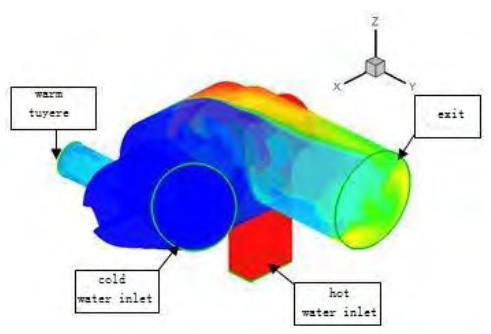

Figure 7. Three-dimensional distribution of flow field temperature



Figure 8. Sectional pressure distribution

It can be seen in the pressure distribution of figure 8 that the high pressure of the warm wind pipe node would not only produce a certain pressure loss, but also make the coolant flow inadequate, affecting the heat transfer of the temperature sensing body. So we could make a simple improvement at here. It was moving the warm wind pipe to the central body or moving it to the same axial direction with cold water inlet. This would make the water of the warm wind pipe in uniform flow and heat transfer easily, as shown in Fig .9.

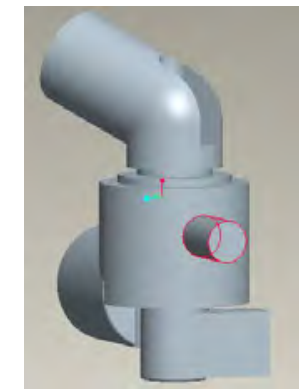

a. improvement scheme 1

Figure 9. Improved design of the warm wind pipe

\section{CONCLUSIONS}

The state of the coolant through the thermostat inner was reflected clearly and intuitively by the visual graphics of flow field inside the thermostat with the CFD simulation analysis. The structure of the thermostat could be appropriately improved or optimized by the temperature, pressure, turbulent kinetic energy and other factors.

Through the simulation in the flow field of the thermostat at different opening, it could be obtained that the thermostat internal fluid flow state and its variation in different operating conditions. It had important significance on the thermostat working law prediction and the thermostat cooling system performance analysis. Since the all above analyses were assumed that the car was running at the same revolving speed, and the impact of the entire cooling system were not considered. The analysis results may be slightly different from the actual situation. If the follow-up works were combined with systems, the research results would be better.

\section{ACKNOWLEDGMENT}

Many people have made invaluable contributions, both directly and indirectly to my research. I would like to express my warmest gratitude to Yan $\mathrm{Li}$, my supervisor, for his instructive suggestions and valuable comments on the writing of this thesis. Without his invaluable help and generous encouragement, the present thesis would not have been accomplished. At the same time, I am also grateful to Prof. Mingshun Yang for providing me with valuable advice and access to the related resources on my thesis.

Finally, I greatly appreciate my parents' support and endless love. My heart swells with gratitude to all the people who helped me.

\section{REFERENCES}

[1] Peng Liu, "Research and Development on the Aerodynamic Thermostat," Wuhan, Wuhan University of Science and Technology, 2008.

[2] T. Mitchell, M. Salah, J. Wagner, and D. Dawson, "Automotive Thermostat Valve Configurations: Enhanced Warm-Up Performance,” Journal of Dynamic Systems, Measurement, and Control.2009, vol. 131, pp. 1-7.

[3] Jiarui Chen, Jianwen Zhang, Automobile Structure. Beijing China Machine Press, 2006

[4] Pan Ding, Weiqi Song, "Discussion on The Thermostat's Development," Industrial Technology, 2012, vol. 36(2), pp. 5657.

[5] Xiaobei Cheng, Li Pan, and Hongling Ju, "Research Progress of Cooling System for Modern Vehicle Engine," Vehicle Internal Combustion Engine, 2008, vol. 1, pp. 1-6.

[6] Shiquan He, "Numerical Simulation and Optimization of the Flow Field of the Self Operated Thermostatic Valve," Fluid Machinery, 2010, vol. 38(4), pp. 29-31.

[7] Fujun Wang, Computational fluid dynamics analysis CFD Software Principles and Applications. Beijing: Tsinghua University Press, 2004

[8] Jianxi Luo, Yangjun Zhang, "Influences of thermostat on engine thermal system transient performance," Journal of Tsinghua University (Science and Technology), 2004, vol. 44, pp. 32-36.

[9] John R. Wagner, Darren M. Dawson, and Egidio Marotta, "An Advanced Engine Thermal Management System: Nonlinear 
Control and Test," TRANSACTIONS ON MECHATRONICS, 2005, vol. 10(2), pp. 210-220.

[10] Shan Jiang, "Three Dimensional Numerical Simulation of Flow Fields Inside Sea Suction Valve," China Ship Research, 2009, vol. 4(2), pp. 38-42.
[11] Yongcheng Pan, "Analysis of Inner Flow Field Characteristics of a Control Valve Based on CFD," Machine Tool and Hydraulics, 2011, vol. 39(1), pp. 1-3. 